\title{
SPLITTING IN MAP GROUPS
}

\author{
LEWIS ROBERTSON AND THEODORE W. WILCOX
}

\begin{abstract}
Every locally compact maximally almost periodic group $G$ has a normal vector subgroup, the centralizer of which is of finite irdex. This vector subgroup is nontrivial whenever the identity component of $G$ is not compact. Furthermore, if $G$ has relatively compact conjugacy classes, then $G \cong \boldsymbol{R}^{n} \times L$ where $L$ has a compact open normal subgroup. Several structure theorems are also obtained for cases in which splitting need not occur.
\end{abstract}

Recall that a locally compact maximally almost periodic, [MAP], group is one which has sufficiently many continuous finite-dimensional unitary representations to separate points. The classical theorem upon which this work is based is the following:

THEOREM (FREUDENTHAL-WEIL). Let $G$ be a connected locally compact group. Then $G$ is MAP if and only if $G$ is a direct product of a vector group and a compact group.

In $\S 1$, it is shown that a [MAP]-group $G$ has a normal vector subgroup, the centralizer of which has finite index in $G$. Furthermore, a general splitting theorem of the Freudenthal-Weil type is proved. In $\$ 2$, some structural results are obtained for [MAP]-groups in which the vector group need not split.

The following notation for certain classes of topological groups was established in [2].

IN-the groups contain a compact neighborhood of $e$ invariant under the action of the inner automorphisms.

SIN-(read "small invariant neighborhoods") the groups have a basic system at $e$ of compact invariant neighborhoods.

FC-the groups have finite conjugacy classes.

$[\mathrm{FC}]_{\mathscr{B}}^{-}$-the groups are locally compact and have relatively compact $\mathscr{B}$-conjugacy classes, where $\mathscr{B}$ is a subgroup of the automorphism group. That is, $G \in[\mathrm{FC}]_{\mathscr{D}}^{-}$means $\{x \in G: \exists \beta \in \mathscr{B}, \beta x=y\}^{-}$is compact for each $y \in G$.

Received by the editors January 4, 1971.

AMS 1970 subject classifications. Primary 22D05, 22D10.

Key words and phrases. Maximally almost periodic, finite-dimensional unitary representation, MAP-groups, [FC]-groups, splitting.

(c) American Mathematical Society 1972 
In general, the use of brackets in this notation indicates that the groups considered are locally compact and a subscript indicates that the notion is being modified relative to some subgroup of the topological automorphism group.

Finally, we denote the group of topological automorphisms of $G$ by $\mathfrak{A}(G)$ and the connected component of the identity of $G$ by $G_{0}$. If $G$ is a locally compact abelian group, then $\hat{G}$ is the character group of $G$. If $H \subset G$ is a subgroup, then $H^{\perp}=\{\chi \in \hat{G}: \chi(h)=1$, all $h \in H\}$ is the annihilator of $H$ in $\hat{G}$. All groups considered will be Hausdorff.

1. Let $G$ be a locally compact abelian group and let $\mathfrak{B}$ be a subgroup of $\mathfrak{A}(G)$. For each $\beta \in \mathfrak{B}$ and $\chi \in \hat{G}$, define $\beta^{*}(\chi)=\chi \circ \beta^{-1} \in \hat{G}$. Let $\mathfrak{B}^{*}=$ $\left\{\beta^{*}: \beta \in \mathfrak{B}\right\}$, so that $\mathfrak{B}^{*} \subset \mathfrak{U}(\hat{G})$ and, in fact, it is well known (compare [3, Theorem 26.9]) that the map $\beta \rightarrow \beta^{*}$ is a topological isomorphism of $\mathfrak{B}$ onto $\mathfrak{B}^{*}$. By $F\left(\hat{G}, \mathfrak{B}^{*}\right)$ we denote the set of characters $\chi$ in $\hat{G}$ for which $\left\{\beta^{*} \chi: \beta^{*} \in \mathfrak{B}^{*}\right\}$ is finite. We note that this is a slight change from the notation of [8]. It is easy to see that $F\left(\hat{G}, \mathfrak{B}^{*}\right)$ is a subgroup of $\hat{G}$. Furthermore, a subgroup $L$ of $\hat{G}$ separates points in $G$ if and only if $L$ is dense in $\hat{G}$. Thus the following formulation of [8, Theorem 1] is valid.

PROPOSITION 1. Let $H$ be a normal locally compact abelian subgroup of a topological group $G$. Let $\mathfrak{B}$ be the subgroup of the group of topological automorphisms of $H$ consisting of the restrictions to $H$ of inner automorphisms of $G$. Then $F\left(\hat{H}, B^{*}\right)$ is a dense subgroup of $\hat{H}$ if $G \in M A P$.

The following theorem is a variant of a result which was previously announced [7, Theorem 4]. We note that in Proposition 1 and Theorem 1, it is not necessary to assume that $G$ be locally compact.

THEOREM 1. Let $G \in M A P$ and let $H$ be a normal subgroup of $G$ such that $H=R^{n} \times T^{m}$ where $n$ and $m$ are nonnegative integers. Then the centralizer, $C(H)$, of $H$ has finite index in $G$.

Proof. We identify $\hat{H}$ with $R^{n} \times Z^{m}$ so that, in the notation of Proposition $1, F\left(\hat{H}, B^{*}\right)$ is a dense subgroup of $R^{n} \times Z^{m}$. Each topological automorphism $\beta^{*}$ in $\mathfrak{B}^{*}$ can be extended linearly to a continuous homomorphism $\beta^{\#}$ of $R^{n+m}$. Furthermore, since the rationals are dense in the reals, each $\beta^{\#}$ is a linear operator on $R^{n+m}$. Let $\mathfrak{B}^{\#}$ be the collection of linear operators so obtained. Clearly the points of $R^{n+m}$ which are finitely orbited by $\mathfrak{B}^{\#}$ form a dense subset of $R^{n+m}$ and hence must contain a basis. If $x$, is an element of this basis, then, since the set $\left\{\beta^{\#} \cdot x_{j}: \beta^{\#} \in \mathfrak{B}^{\#}\right\}$ is finite, there can only be finitely many $j$ th columns in the matrix representations of the $\beta^{\#}$. It follows that $\mathfrak{B}^{\#}$, hence $\mathfrak{B}^{*}$ and $\mathfrak{B}$ are all finite. On the other hand, the cardinality of $\mathfrak{B}$ equals $[G: C(H)]$. That is, the centralizer of $H$ in $G$ has finite index in $G$. 
We wish to prove that there are normal vector subgroups in locally compact MAP groups and for this purpose we require the following:

LEMMA 1. Let $G$ be a locally compact abelian group which is the direct product of an n-dimensional vector group $N$ and a compact group $C$. Suppose that $\left\{E_{i}: i \in I\right\}$ is a descending chain of closed subgroups such that $E_{i} /\left(E_{i} \cap C\right)$ is an n-dimensional vector group. Let $E=\bigcap\left\{E_{i}: i \in I\right\}$. Then $E /(E \cap C)$ is an n-dimensional vector group.

Proor. This lemma was first proved in [4, 9.3 Lemma, p. 34]. The following is a shorter and simpler proof. Since $G$ is the direct product of $N$ and $C$, we have $\hat{G}=C^{\perp} N^{\perp}$, where $C--R^{n}$ and $C^{\perp}$ is open in $\hat{G}$. Note that $C$ being compact implies that $E_{i} C / C \cong E_{i} /\left(E_{i} \cap C\right) \cong R^{n}$. It follows that $G=E_{i} C$. That is, $E_{i}^{\perp} \cap C^{\perp}=\{e\}$ so that $E_{i}^{\perp} \cap\left(C^{\perp}-\{e\}\right)=\varnothing$. Thus $E^{\perp}=$ $\left(\cup E_{i}^{\perp}\right)^{-}$does not meet the open set $C^{\perp}-\{e\}$. Consequently, $E^{\perp} \cap C^{\perp}=\{e\}$ and $G=E C$. Thus $R^{n} \cong G / C=E C / C \cong E /(E \cap C)$.

THEOREM 2. Let $G \in[M A P]$. Then $G$ contains a normal vector subgroup $V$ of dimension $n$. This $n$ equals the dimension of the vector component of the connected component of the identity of $G$.

Proof. Let $H$ be the connected componentof the center of the connected component of $G$. That is, $G \supset G_{0} \supset Z\left(G_{0}\right) \supset\left(Z\left(G_{0}\right)\right)_{0}=H$. Since $H$ is a connected locally compact abelian group, $H=N C$ is the direct product of an $n$-dimensional vector group $N$ and a compact connected group $C$. Furthermore, it is easy to see that this $n$ equals the $n$ in the statement of the theorem and that $C$ is the unique maximal compact subgroup of $H$. Let $\mathbb{C}$ be the collection of subgroups $E$ of $H$ such that

1. $E$ is a closed normal subgroup of $G$ and

2. $E /(E \cap C)$ is an $n$-dimensional vector group.

We note that $\mathbb{C}$ is nonempty since $H$ is a characteristic subgroup of $G$ and consequently $H \in \mathbb{C}$. By Zorn's lemma and Lemma 1 , there is a minimal element $E$ in $C$ where $E=V A$ is a direct product of an $n$-dimensional vector group $V$ and a compact abelian group $A$. Furthermore, $A$ is connected for otherwise $E_{0}=V A_{0}$ would be a proper characteristic subgroup of $E$ and the quotient of $E_{0}$ by the subgroup $A_{0}=E_{0} \cap C$ of compact elements of $E_{0}$ would be an $n$-dimensional vector group which would contradict the minimality of $E$ in $\mathbb{C}$.

If $A=\{e\}$, we are done. Thus we assume that $A$ is nontrivial and will arrive at a contradiction. Since $G \subseteq[\mathrm{MAP}]$, there is a representation $\Pi$ of $G$, that is, a continuous homomorphism $\Pi: G \rightarrow U$ where $U$ is a (unitary) Lie group, such that $\Pi(A) \neq\{I\}$. Let $K=A \cap \operatorname{ker} \Pi$. Then $K$ is a normal subgroup of $G$ since $A$ is a characteristic subgroup of the normal subgroup $E$. Furthermore, $A / K \cong \Pi(A)$ is a connected compact abelian Lie group; 
that is, $A / K \cong T^{m}$ for some positive integer $m$. It follows that $E / K$ is a normal subgroup of $G / K$ and that $E / K \cong R^{n} \times T^{m}$. Since $G \in[\mathrm{MAP}]$ and $K$ is compact, we have $G / K \in[\mathrm{MAP}]$. (See [5, Proposition 1].) By Theorem 1, the centralizer of $E / K$ has finite index in $G / K$. Thus the group of automorphisms of $E / K$ which are the restrictions to $E / K$ of inner automorphisms of $G / K$ is finite. By [1, Lemma 1] or [4, Theorem X] there is a closed subgroup $W / K$ of $E / K$ with $W / K \cong R^{n}$ which is invariant under the action of this finite group of automorphisms. It follows that $W$ is a proper subgroup of $E, W$ is a normal subgroup of $G$, and $K=W \cap C$. Thus $W \in \mathbb{C}$ which contradicts the choice of $E$.

THEOREM 3. Let $G \in[M A P] \cap[F C]^{-}$. Then $G \in[S I N]$. Furthermore, $G \cong V \times H$ where $V$ is a vector group, $H \in[F C]^{-} \cap[M A P]$, and $H$ contains $a$ compact open normal subgroup $K$.

Proof. Let $P$ be the set of those elements of $G$ which are contained in compact normal subgroups of $G$. By the structure theorem for $[\mathrm{FC}]^{-}$groups [2, Theorem 3.16], we know that $P$ is a closed normal subgroup of $G$ and that $G / P$ is a direct product of a vector group and a discrete group $D$. Let $V$ be the normal vector subgroup of $G$ obtained in Theorem 2 . Since the connected component of the identity in $G$ is a direct product of $V$ with a compact group, it follows that the connected component, $(G / P)_{0}$, of $G / P$ is $V P / P$. Thus we have the following composition of maps

$$
G \stackrel{\pi_{1}}{\longrightarrow} G / P=V P / P \times D \stackrel{\pi_{2}}{\longrightarrow} V P / P \stackrel{\varphi}{\longrightarrow} V
$$

where $\varphi$ is a topological isomorphism, $\varphi(v P)=v$ for $v \in V$. Clearly the above composition is a continuous projection of $G$ onto $V$ so that $G \cong V \times H$. It is known that every $[\mathrm{FC}]^{-}$group has an invariant neighborhood. (For a proof see Corollary 2.2 of Dual spaces of locally compact groups with precompact conjugacy classes by J. Liukkonen which will soon appear in Trans. Amer. Math. Soc.) That is, $G \in[\mathrm{IN}]$. Since [MAP] $\cap[\mathrm{IN}] \subset[\mathrm{SIN}]$ (see [4, Proposition 12.2]), $G$ has small invariant neighborhoods and it follows that $H$ contains a compact open normal subgroup $K$.

2. The material in this section is motivated by the following example. Let $G$ be the group of all matrices

$$
\left(\begin{array}{ccc}
1 & n & x \\
0 & \vdots & m \\
0 & 0 & 1
\end{array}\right), \quad n, m \in Z, x \in R
$$

and let $H$ be the copy of the real line in $G$ consisting of the above matrices when $n=m=0$. Then $G$ is a compactly generated [MAP] group (thus also 
in [SIN]), $H$ is the connected component, the center and the commutator subgroup of $G$, and $G / H \cong Z \times Z$ but $H$ does not split in $G$. This is essentially Example 11.10 of [4]. We ask about the extent that this example is typical of the situation when splitting does not occur.

The following theorem is a modification of [2, Proposition 4.5].

THEOREM 4. Let $G$ be a compactly generated group in [MAP]. Assume that $G / G_{0}$ is in $[F C]^{-}$. Then $G$ contains an open normal subgroup $H$ such that

(1) $H=V K$ is a semidirect product of a vector group $V$ normal in $G$ ana a compact group $K$ and

(2) $G / H \cong Z^{n}$ for some nonnegative integer $n$.

Proof. We recall that a compactly generated [MAP] group is in [SIN] [4, Proposition 12.2 (iv)]. Since $G \in[\mathrm{MAP}] \cap$ [SIN], by Theorem 2, $G$ contains a normal vector group $V$ and an open normal subgroup $N$ such that $N / V$ is compact. By Iwasawa's theorem (see e.g., [4, Proposition 7.6]) $N=V C$ is a semidirect product of $V$ with a compact group $C$. Then $G^{*}=$ $G / N$ is a finitely generated (discrete) FC group. By [6, Theorem 5.1] the commutator subgroup of $G^{*}$ is finite so that $G^{*}$ contains a finite normal subgroup $F^{*}$ such that $G^{*} \mid F^{*} \cong Z^{n}$. Let $\pi: G \rightarrow G / N=G^{*}$ be the natural projection and let $H=\pi^{-1}\left(F^{*}\right)$. Since $H / N \cong F^{*}$ is finite (compact) and $N / V$ is compact, $H / V$ is compact. By Iwasawa's theorem again, we have $H=V K$ is a semidirect product with $K$ compact. This concludes the proof.

We should note that by $[2$, Proposition 4.10$]$ the above hypotheses imply that $G_{i}^{\prime} G_{0}$ is in [MAP].

COROLlaRY 1. Let $G$ be a compactiy generated group in [MAP]. Assume that $G / G_{0}$ is abelian. Then $G$ contains an open normal subgroup $H$ such that

(1) $H=V K$ is a direct product of a vector group $V$ normal in $G$ and a compact group $K$ and

(2) $G / H$ is a finitely generated abelian group.

Proof. Except for the statement that $V$ is normal in $G$, this is an obvious consequence of the characterization of [SIN] groups [2, Theorem 2.13]. The full statement is obtained from Theorem 4 as follows. Let $H_{1}=$ $V K_{1}$ be the subgroup guaranteed by Theorem 4. By Theorem 1 , for example, $K_{1}$ contains an open normal subgroup $K$ of finite index such that $H=V K$ is a direct product. Since $H$ contains $G_{0}$ and $G / G_{0}$ is abelian, $H$ is normal in $G$. Clearly $G / H$ is both finitely generated and abelian.

COROLlARY 2. Let $G$ be a compactly generated group in [MAP]. Let $V$ be the normal vector subgroup of $G$ whose existence is guaranteed by Theorem 2 and assume that $G / V$ is abelian. Then $G=L K$ is a semidirect 
product of a compact (abelian) group $K$ with a normal subgroup $L$ containing $V$ such that $L / V \cong Z^{n}$ for some nonnegative integer $n$.

Proof. Since $G / V$ is a compactly generated abelian group with no vector component, $G / V=(L / V)(F / V)$ is a direct product with $L / V \cong Z^{n}$ for some $n$ and $F / V$ compact. Furthermore by Iwasawa's theorem $F=$ $V K$ is a semidirect product of $V$ with a compact group $K$. We wish to show that $K$ splits in $G$. A projection of $G$ onto $K$ is given by the following composition:

$$
G \stackrel{\pi_{1}}{\longrightarrow} G / V=(L / V)(F / V) \stackrel{\pi_{2}}{\longrightarrow} F / V=(V K) / V \stackrel{\varphi}{\longrightarrow} K /(K \cap V)=K .
$$

Here $\pi_{1}$ and $\pi_{2}$ are natural projections and, since $K$ is compact, the natural isomorphism $\phi$ is also a homeomorphism [3, Theorem 5.33]. Thus if $k \in K$, we have $q \pi_{2} \pi_{1}(k)=\varphi \pi_{2}(k V)=q(k V)=k$. By [4, Theorem 1, p. 8] it follows that $G$ is a semidirect product of $L$ with $K$.

\section{REFERENCES}

1. S. Grosser and M. Moskowitz, On central topological groups, Trans. Amer. Math. Soc. 127 (1967), 317-340. MR 35 \#292.

2. - Compactness conditions in topological groups. I, II, J. Reine Angew. Math. 146 (1971), 1-40.

3. E. Hewitt and K. A. Ross, Abstract harmonic analysis. Vol. 1: Structure of topological groups. Integration theory, group representations, Die Grundlehren der math. Wissenschaften, Band 115, Academic Press, New York; Springer-Verlag, Berlin, 1963. MR 28 \#158.

4. K. H. Hofmann and P. Mostert, Splitting in topological groups, Mem. Amer. Math. Soc. No. 43 (1963). MR 27 \#1529.

5. H. Leptin and L. Robertson, Every locally compact map group is unimodular, Proc. Amer. Math. Soc. 19 (1968), 1079-1082. MR 37 \#6397.

6. B. H. Neumann, Groups with finite classes of conjugate elements, Proc. London Math. Soc. (3) 1 (1951), 178-187. MR 13, 316.

7. T. W. Wilcox, On the structure of maximally almost periodic groups, Bull. Amer. Math. Soc. 73 (1967), 732-734. MR 35 \#4344.

8. - On the structure of maximally almost periodic groups, Math. Scand. 23 (1968), 221-232. MR 40 \#5788.

Department of Mathematics, Wesleyan University, Middletown, Connecticut 06457 (Current address of Lewis Robertson)

Department of Mathematics, University of Rochester, Rochester, New york 14627

Current address (Theodore W. Wilcox): Mathematisches Institut der Technischer Universität, (8) Munchen 2, Arcisstrasse 21, Federal Republic of Germany 